\title{
Korteweg-de Vries characters in large central charge CFTs
}

\author{
Enrico M. Brehm* \\ Max Planck Institut für Gravitationsphysik, Albert-Einstein-Institut, Potsdam-Golm D-14476, Germany \\ Diptarka Das $\circledast^{\dagger}$ \\ Indian Institute of Technology, Kanpur, Kanpur, Uttar Pradesh-208016, India
}

(Received 10 October 2019; accepted 15 April 2020; published 27 April 2020)

\begin{abstract}
Two-dimensional conformal field theories with just Virasoro symmetry are endowed with integrable structure. We review how to construct the integrable charges in a two-dimensional conformal field theory and how to relate them to the charges of quantum sinh-Gordon theory when $c \geq 25$. We then explicitly calculate the single charge characters in the large $c$ limit for all charges and thereby reveal how their degeneracies grow within one module. This, in particular, allows us to approximate the characters in the limit of small chemical potential, which source the respective charges. The latter gives us insights into possible transformation properties of the characters. We also comment on the full generalized Gibbs ensemble and approximations to pure states.
\end{abstract}

DOI: 10.1103/PhysRevD.101.086025

\section{INTRODUCTION}

At the heart of th solvability of two-dimensional (2D) conformal field theories (CFTs) lies the infinite dimensional Virasoro algebra. This permits the construction of an infinite set of mutually commuting charges, first exhibited in [1] and also [2]. A deep connection between CFTs and integrable theories arises when quantizing soliton equations (specifically, the sine-Gordon equation). The charges themselves are constructed as integrals over (finite) polynomials of the energy momentum tensor and as such are polynomial functions of the Virasoro generators. Often one calls them the Korteweg-de Vries (KdV) charges of the conformal field theory, $\hat{K}_{n}$, since they are classically related to the integrals of motion of the $\mathrm{KdV}$ equation [see (15)]. These integrable nonlinear partial differential equations (PDEs) appear naturally as equations of motion for the sine-Gordon as well as the sinh-Gordon theories. For the $c \leq 1$ case, one can relate the infinite set of these charges in the CFT to the KdV charges of the sine-Gordon theory [1]. It turns out that there exists a modification of the analysis which relates the charges in large $c$ CFTs to the sinhGordon theory. In this work we will be concerned with various properties of $\mathrm{KdV}$ charges in large $c$ CFTs;

\footnotetext{
*brehm@aei.mpg.de

†didas@iitk.ac.in
}

Published by the American Physical Society under the terms of the Creative Commons Attribution 4.0 International license. Further distribution of this work must maintain attribution to the author(s) and the published article's title, journal citation, and DOI. Funded by SCOAP ${ }^{3}$. therefore as a first step we explicitly carry out the relevant analytic continuations that relate a $c \geq 25$ CFT to the sinhGordon model. ${ }^{1}$

All the infinite $\mathrm{KdV}$ charges commute with the Hamiltonian of the CFT, $\left[H_{\mathrm{CFT}}, \hat{K}_{n}\right]=0$. Therefore they generate a rigid infinite dimensional Abelian symmetry within the larger symmetry generated by the full enveloping Virasoro algebra. Due to their connection to the theory of integrable systems one can apply techniques thereof in the context of CFT. This was done in the series of remarkable papers [3-5], where they map the problem of finding the exact eigenvalues of all these charges in the $c \leq 1$ CFTs to that of solving the pure quantum mechanical problem of a single particle in a specific potential. Moreover, under specific relevant perturbations, the integrability structure is retained. This has been utilized to find the exact $S$-matrix off-criticality $[6,7]$ and thereby provides additional motivation to understand the charges in CFTs.

The KdV charges can also be used to define a generalized partition function, or the generalized Gibbs ensembles (GGE). When a critical system is in equilibrium with some environment, such partition functions naturally arise. If we do not specify the allowed interactions between the system and the environment, then one needs to take all conserved quantities into account. Such a generic equilibrium state of the system with chemical potentials for every possible conserved quantity can be written

\footnotetext{
${ }^{1}$ Without this connection being made, it is a priori unclear whether the infinite set of charges in large $c$ CFTs is related to the $\mathrm{KdV}$ integrable hierarchy.
} 


$$
\rho\left(\left\{q, y_{i}\right\}\right)=q^{H_{\mathrm{CFT}}} \prod_{i \geq 2} y_{i}^{\hat{K}_{i}},
$$

where $q=e^{-\beta}$ with the inverse temperature $\beta$, and $y_{i} \equiv e^{-\mu_{i}}$ with the chemical potential $\mu_{i}$. The GGE itself is then given by

$$
\begin{aligned}
Z\left(\left\{q, y_{i}\right\}\right) & =\operatorname{Tr}\left[\rho\left(\left\{q, y_{i}\right\}\right)\right] \\
& =\operatorname{Tr}_{[0]}\left[\rho\left(\left\{q, y_{i}\right\}\right)\right]+\sum_{p} \operatorname{Tr}_{[p]}\left[\rho\left(\left\{q, y_{i}\right\}\right)\right],
\end{aligned}
$$

where the first trace is over the full spectrum of the CFT, and $\operatorname{Tr}_{[p]}$ stands for the trace over a primary module $[p]$, with [0] denoting the vacuum module. The subtraces over specific modules will naturally be called the GGE characters.

Purely thermal states are obviously given by $\rho\left(\left\{q, y_{i}=1\right\}\right)$, i.e., all chemical potentials have to vanish. In two-dimensional CFT, the thermal ensemble-or partition function-has a nice geometric description. If the theory is defined on a circle of length $L$, then the thermal ensemble is the partition function of the theory on a rectangular torus with modular parameter $\tau=i \beta / L$. Furthermore, if one demands that the CFT is well defined on a torus, then its partition function must be invariant under modular transformations

$$
\tau \mapsto \frac{a \tau+b}{c \tau+d}, \quad\left(\begin{array}{ll}
a & b \\
c & d
\end{array}\right) \in \operatorname{PSL}(2, \mathbb{Z}) .
$$

One of its generators is the so-called $S$-transformation $\tau \mapsto-1 / \tau$. On the level of temperature, this means that the partition function must be invariant under $\beta \mapsto \frac{L^{2}}{\beta}$ which directly relates different temperature regimes, in particular the partition function at a very low temperature, where it is basically given by the vacuum result, to the very high temperature partition function, where high energy states dominate. This allows one to bootstrap the theory and compute universal results on the growth of its high energy spectrum [8,9]. One can also bootstrap the CFT by considering correlation functions on the torus, which are not invariant but transform covariantly under modular transformations. This allows one to obtain universal information about asymptotic matrix elements/operator product expansion (OPE) coefficients in 2D CFT [10-12].

A natural question while considering GGEs is whether similar features arise for the $\mathrm{KdV}$ charges. Namely, is there a transformation $y_{i} \mapsto \tilde{y}_{i}$ which relates the different regimes of the GGE and correlation functions in the corresponding state? If such a transformation exists, can it be used to bootstrap the CFT? A related second order question is whether there is a geometrical description for GGEs such that $y_{i}$ plays the role of moduli. A motivation for this expectation comes from considering classical integrable systems. Riemann surfaces play an ubiquitous role in the analytic study of integrable systems. Solutions of the integrable hierarchy are related to finding sections of an eigenvector bundle over a Riemann surface [13]. The hope is that this classical connection also reflects itself in the GGE of the quantum theory.

Considerations of the GGE as a possible generalization of thermalization of pure states and in particular the eigenstate thermalization hypothesis (ETH) also provides yet another strong motivation. It is widely believed that in many nonintegrable quantum theories generic pure states look approximately thermal after waiting long enough. This, in particular, implies that static states, i.e., energy eigenstates, can be approximated by thermal states. The question arising here is whether generic pure states in integrable systems might be approximated by GGE states, rather than thermal states, when waiting long enough. This then implies that generic energy eigenstates would always look like states in equilibrium with respect to all the conserved charges of the system.

In this article we take small steps in the direction of addressing the above questions. In particular, we compute the GGE characters in the large central charge limit. Recently in [14-16], aspects of GGE were studied in the regime where the chemical potentials are parametrically smaller than the inverse temperature, which itself is small enough to probe energies much larger than the central charge. Our analysis is in a complementary regime, which means $\mu_{l} \gg 1 /\left(c^{1-\frac{1}{2 l}}\right), \beta \gg 1 / \sqrt{c}$, and we normally take $\mu_{l} \gg \beta$. Large $c$ is also the classical limit of the corresponding soliton theory, and the integrable charges can be computed by a relatively easy recursive formula. In this limit it is possible to diagonalize all charges simultaneously and give their eigenvalues in a closed form. This allows us to give good estimates on the large and small chemical potential regimes, such that possible transformations are tractable.

The article is organized as follows. In Sec. II we review the connection between quantum sinh-Gordon theory and conformal field theory. Following [1] we explicitly demonstrate the analytical continuation to central charges $c>1$. Our analysis reveals that the Feign-Fuchs form of the energy momentum tensor, which is needed to draw the connection to quantum integrable theory, is closely related to the bound state sectors of quantum Liouville theory that describes two-dimensional nonperturbative gravity. We will hopefully deepen the understanding of this connection in future work. In Sec. III we compute the GGE characters in mixed states with a single nonzero chemical potential at a parametrically large central charge. We first concentrate on the second integrable charge and then generalize to higher ones. We again want to point out that our calculation is valid in regimes, where the chemical potentials are such that the eigenvalues that contribute dominantly to the GGE character are small compared to the central charge. In the following Sec. IV we comment on possible transformations 
that relate regimes of high and low chemical potentials. For this we analyze how the charge degeneracy grows within a module and perform the sum over the vacuum module for large charges by an integral evaluated via the saddle point approximation. We also compare the result to the full GGE at parametrically small chemical potentials as considered in $[14,15,17]$. In both cases it shows qualitatively the same behavior. In particular, this also matches the well-known results of thermal states and might indicate that there indeed is some transformation that relates high and low chemical potentials. However, the results show that transformation of the chemical potentials of higher integrable charges cannot simply be the standard modular transformations, and it is not clear to us if there might exist a geometric interpretation. In the following Sec. V we shortly comment on the possible generalization of ETH in the presence of integrable charges. In Sec. VI we conclude on our findings. In the appendixes we give specific formulas to compute the classical and quantum KdV charges, give some explicit checks on the large $c$ eigenvalues of the second $\mathrm{KdV}$ charge, and compute the global character of the second charge which appears as a factor of the GGE characters in a primary module.

\section{FROM CFT TO QUANTUM SINH-GORDON THEORY}

The connection between conformal and integrable field theories has a long history. We want to go back to some of the earliest works and briefly review how one can connect two-dimensional conformal field theories with models such as the sinh-Gordon theory [1]. Although the integrable charges themselves can be constructed for any 2D CFT the latter connection is not always present. However, only with this connection does it become clear why large $c$ indeed is a classical limit and how the quantum integrable structure is related to the classical integrable structure. This connection is what gives the idea to search for a geometric meaning of the chemical potentials. In what follows we do, however, not concentrate on the $c<1$ case as in [1], but do concentrate on an analytic continuation to $c>1$. In fact, it turns out that the theories we have to consider have $c \geq 25$.

Let us consider some conformal field theory and assume that we can write its energy momentum tensor in FeignFuchs (or Coulomb-gas) form

$$
T^{+}(z) \equiv \frac{1}{\hbar}\left(\frac{1}{2}:(D \phi)^{2}:-\lambda D^{2} \phi+\frac{\lambda^{2}}{2}\right),
$$

where $\lambda$ is some real constant, $D=i z \partial_{z}$ with $z$ the compactified coordinate on the plane, $z=e^{\tau+i \sigma}$, and

$$
\phi(z)=i q-i \alpha_{0} \log (z)+i \sum_{n \neq 0} \frac{\alpha_{n}}{n} z^{-n}
$$

is a generalized free field whose modes obey the standard commutation relations

$$
\left[\alpha_{n}, \alpha_{m}\right]=\hbar n \delta_{n+m}, \quad\left[q, \alpha_{n}\right]=i \hbar \delta_{n}
$$

One can show that this energy momentum tensor corresponds to a Virasoro algebra with central charge $c=1+\frac{12 \lambda^{2}}{\hbar}$. Its generators can be written as

$$
\hbar L_{n}^{+}=\frac{1}{2} \sum_{p}: \alpha_{p} \alpha_{n-p}:+i \lambda n \alpha_{n}+\frac{\lambda^{2}}{2} \delta_{n} .
$$

We want to follow the logic of [1] and define a second energy momentum tensor as

$$
T^{-}(z) \equiv \frac{1}{\hbar}\left(\frac{1}{2}:(D \phi)^{2}:+\lambda D^{2} \phi+\frac{\lambda^{2}}{2}\right) .
$$

Hermitian conjugation is given by $\left(L_{n}^{+}\right)^{\dagger}=L_{-n}^{-}$, for which one consistent choice that we can make is the relation $\alpha_{n}^{\dagger}=-\alpha_{-n}$. Note that by this we lose the usual direct connection between asymptotic in and out states of the quasi-free field $\phi$.

We now shortly want to comment on the similarities of the present construction to Liouville theory. Its energy momentum tensor in a strip can actually be written exactly as in (4) where the generalized field $\phi$ can be expressed in terms of the Liouville field $\varphi$ (see e.g., [18]). When considering bound states in the Liouville theory a simple connection between in and out states by Hermitian conjugation, as in the case of scattering states, is now no longer possible [18]. It seems reasonable that the integrable structure that we want to present here is also visible in the transition amplitudes of bound states in Liouville theory on a strip.

Let us now consider the dressed Vertex operators

$$
V^{ \pm}=z^{-\beta \lambda}: e^{ \pm \beta \phi}:, \quad \beta \in \mathbb{R}
$$

with conformal weight $h=-\frac{\hbar}{2} \beta^{2}+\beta \lambda$ with respect to $T^{ \pm}$, respectively. Now, to draw the connection between the CFT and the quantum sinh-Gordon one needs $h=1$ as we will see later. The secondary field $z^{h} V^{ \pm}$then naturally converges to $e^{ \pm \beta \phi}$ in the classical limit $\hbar \rightarrow 0$. Therefore, the operator

$$
\hat{H}=\frac{1}{2 \pi i \beta^{2}} \oint \frac{d z}{z}\left(1-\frac{1}{2}\left(z^{1-\beta \lambda}: e^{\beta \phi}:+z^{1-\beta \lambda}: e^{-\beta \phi}:\right)\right)
$$

converges for $\hbar \rightarrow 0$ to the classical Hamiltonian of sinhGordon theory 


$$
H=\frac{1}{2 \pi \beta^{2}} \int_{0}^{2 \pi} d \sigma(1-\cosh \beta \phi),
$$

which, with the identification $u=\frac{\beta}{2} \partial_{\sigma} \phi$, canonically generates the sinh-Gordon equation ${ }^{2}$

$$
\partial_{t} \partial_{\sigma} \phi=\frac{2}{\beta} \partial_{t} u=\{u, H\}=-\frac{1}{\beta} \sinh \beta \phi .
$$

An important point now is that there exists an infinite set of polynomial conserved quantities $I_{n}$, defined in (A1), which is the same as for the modified Korteweg-de Vries $(\mathrm{mKdV})$ equation

$$
u_{t}=u_{\sigma \sigma \sigma}-6 u^{2} u_{\sigma}
$$

Being a conserved quantity in particular means that $\left\{I_{n}, H\right\}=0$.

Another observation is that the analytic continuation of the classical Miura transform

$$
v=u^{2} \pm u_{\sigma}
$$

maps any solution of the above $\mathrm{mKdV}$ equation to a solution of the $\mathrm{KdV}$ equation

$$
v_{t}=v_{\sigma \sigma \sigma}-6 v v_{\sigma},
$$

which also gives rise to an infinite set of polynomial conserved quantities $K_{n}$ as defined in (A3). They are connected to the conserved quantities of the $\mathrm{mKdV}$ by

$$
K_{n}\left(v=u^{2} \pm u_{\sigma}\right)=I_{n+1}(u) .
$$

It is clear that

$$
\left\{K_{n}(v), H\right\}=0
$$

must be true too.

Let us now come back to quantum theory and the CFT. With the identification $u=\frac{\beta}{2} D \phi$ as in the classical case, the energy momentum tensor of the CFT can be regarded as a quantum Miura transform of that field,

$$
\begin{aligned}
\mathcal{L}^{+}(z) & =\hbar^{\prime}\left(T^{+}(z)+\frac{1-c}{24}\right)=\frac{1}{2}\left(: u^{2}:-\lambda \beta D u\right), \\
\hbar^{\prime} & =\left(\frac{\beta}{2}\right)^{2} \hbar .
\end{aligned}
$$

To see the mKdV hierarchy at quantum level we therefore demand that any Hermitian operator/functional

\footnotetext{
${ }^{2}$ The canonical Poisson bracket needs to be $\left\{\phi(\sigma), u\left(\sigma^{\prime}\right)\right\}=$ $-\beta \pi \delta\left(\sigma-\sigma^{\prime}\right)$.
}

$\hat{F}\left(\mathcal{L}^{+}\right)\left[\hat{F}\left(T^{+}\right)\right]$commutes with the quantum sinh-Gordon Hamiltonian $\left[\hat{F}\left(\mathcal{L}^{+}\right), \hat{H}\right]=0$. We consider functionals of the form

$$
F\left(T^{+}\right)=\frac{1}{2 \pi i} \oint \frac{d z}{z} P^{(n)}\left(\mathcal{L}^{+}, D \mathcal{L}^{+}, \ldots\right),
$$

where $P^{(n)}$ are polynomials of $\mathcal{L}^{+}$and its derivatives. All these functionals in particular commute with $V^{+}$if all the Virasoro generators commute with it,

$$
\left[L_{n}^{+}, z^{h} V^{+}\right]=0 \forall_{n},
$$

which is possible only if the previously defined dressed Vertex operator has unit conformal weight,

$$
h=-\frac{\hbar}{2} \beta^{2}+\beta \lambda=1 \Rightarrow \lambda=\frac{1}{\beta}\left(1+\frac{\hbar}{2} \beta^{2}\right) .
$$

The additional constraint

$$
\left[F\left(\mathcal{L}^{+}\right), z^{h} V^{-}\right]=\left[F\left(\mathcal{L}^{+}\right)^{\dagger}, z^{h} V^{-}\right]=\left[F\left(\mathcal{L}^{-}\right), z^{h} V^{-}\right]=0
$$

is, respectively, solved if for all $n$ we demand $\left[L_{n}^{-}, z^{h} V^{-}\right]=0$ which also gives $h=1$.

With these constraints we, in particular, obtain $c=$ $13+\frac{3}{\hbar^{\prime}}+12 \hbar^{\prime}$, which has a minimal value of $c=25$ at $\hbar^{\prime}=\frac{1}{2}$ and in the classical limit $\hbar^{\prime} \rightarrow 0$ goes as $c \approx \frac{3}{\hbar^{\prime}}$. It also becomes evident that in the classical limit the quantum Miura transformation (18) becomes the classical Miura transformation (14). Note that the precise shift of the energy momentum tensor in (18) is, in fact, needed to preserve the same kind of connection between the $\mathrm{KdV}$ and the $\mathrm{mKdV}$ structures on the quantum level as in the classical case.

Following the logic in constructing the classical charges, one constructs the quantum KdV charges $\hat{K}_{n}$ as functionals (19), i.e., as integrals of polynomials of $\mathcal{L}^{+}(z)$ and its derivatives, that are related to the $\mathrm{mKdV}$ charges of the quantum sinh-Gordon theory by the quantum Miura transform (18) and are constrained as follows:

(i) The charges are Hermitian, $\hat{K}_{n}=\hat{K}_{n}^{\dagger}$.

(ii) The charges commute among each other, $\left[\hat{K}_{n}, \hat{K}_{m}\right]=0$.

(iii) If we assign grade 2 to the field $\mathcal{L}$ and grade 1 to the derivative, then $P^{(n)}$ have total grade $2 n$.

(iv) In the classical limit $\hbar^{\prime} \rightarrow 0\left(c \rightarrow \frac{3}{\hbar^{\prime}}\right)$ the charges reproduce the classical integrals of motion (A3) (when identifying $v=-2 \mathcal{L}$ ).

There is no closed formula known for all charges. However, one can construct them step by step with increasing grade. The first two KdV charges in a CFT are given by

$$
H \equiv \hat{K}_{1}=\frac{1}{2 \pi \hbar^{\prime} i} \oint \frac{d z}{z} \mathcal{L}(z)=L_{0}-\frac{c-1}{24},
$$




$$
\begin{aligned}
Q & \equiv \hat{K}_{2}=\frac{1}{2 \pi \hbar^{\prime} i} \oint \frac{d z}{z} 2 \mathcal{L}^{2}(z) \\
& =2 \hbar^{\prime}\left(2 \sum_{n=1}^{\infty} L_{-n} L_{n}+L_{0}^{2}-\frac{c-1}{12} L_{0}+\frac{(c-1)^{2}}{576}\right) \\
& =Q^{(1)}+2 \hbar^{\prime}\left(L_{0}^{2}-\frac{c-1}{12} L_{0}+\frac{(c-1)^{2}}{576}\right) .
\end{aligned}
$$

More are given in (B5). Note that the charges we give here slightly differ from the charges considered in [3], and also as they were used in $[14,15]$. This is because we strictly follow the conventions of [1] and construct the charges from $\mathcal{L}=T+\frac{1-c}{24}$ to obtain the direct connection to the quantized sinh-Gordon theory. At finite $c$ this has the effect that the charges we use, and are constructed in [1], are some simple linear combinations of the charges used in [3]. We also want to mention that there also exists a discretized construction for the charges as given in [19].

\section{KdV CHARACTERS}

As a first step to understand the full GGE, we start investigating the GGE characters which we define as the trace of a GGE state over a specific highest weight module,

$$
Z_{\left\{q, y_{i}\right\}}^{p}=\operatorname{Tr}_{[p]} \rho\left(\left\{q, y_{i}\right\}\right)
$$

We will analyze this quantity in the classical limit, i.e., when $c \rightarrow \frac{3}{\hbar^{\prime}} \rightarrow \infty$. We also assume that the chemical potentials are not too small which in our case means that $\mu_{i} \gg 1 / c^{1-\frac{1}{2 i}}{ }^{3}$ This probes regimes of the GGE characters for which the corresponding charge is small compared to $c$. In this limit we are able to diagonalize all $\mathrm{KdV}$ charges simultaneously in a simple basis. To do so let us consider the renormalized Virasoro generators (for $n \neq 0$ ) $L_{n} \rightarrow$ $L_{n}^{\prime}=\frac{L_{n}}{\sqrt{c}}$, with

$$
\left[L_{m}^{\prime}, L_{n}^{\prime}\right]=\frac{m^{3}-m}{12} \delta_{m,-n}+\frac{m-n}{\sqrt{c}} L_{m+n}^{\prime} \approx \frac{m^{3}-m}{12} \delta_{m,-n},
$$

where approximations from now on only keep the leading order results at large $c .{ }^{4}$ Now consider states in a primary $p$-module of the form

$$
\left|\left\{n_{i}, k_{i}\right\}\right\rangle=\prod_{i=1}^{M} L_{-n_{i}}^{\prime k_{i}}|p\rangle .
$$

\footnotetext{
${ }^{3}$ This constraint comes from a saddle point analysis and will become clear in (54).

${ }^{4}$ This unusual normalization of the Virasoro generators with $\sqrt{c}$ also appears in [20] where the authors studied the CFT dual of graviton exchanges in the heavy-light conformal blocks.
}

From the above commutation relation it follows that they are orthogonal up to large $c$ corrections. A possible basis is for example the set of all states with $L_{-n_{i}}^{\prime}$ ordered such that bigger $n_{i}$ always appear on the left of smaller $n_{i}$.

All the above states are, in fact, approximate eigenstates of all $\mathrm{KdV}$ charges, in the large $c$ limit. Let us first show this for the second $\mathrm{KdV}$ charges $Q$ as given in (24).

\section{A. Warm-up: $Q$ character at large central charge}

The above states (27) are clearly eigenstates of $L_{0}$ with eigenvalue $\sum_{i=1}^{M} k_{i} n_{i}$. One can show that with the commutation relation (26) it follows that they are also eigenstates of $Q^{(1)}$. This is because only terms from the central extension contribute to order $c^{0}$. The approximate $Q^{(1)}$ eigenvalue of a state $\left|\left\{n_{i}, k_{i}\right\}\right\rangle$ is given by

$$
\lambda_{\left\{n_{i}, k_{i}\right\}} \approx \sum_{i=1}^{M} k_{i}\left(n_{i}^{3}-n_{i}\right)
$$

In Appendix B 3 we provide checks of the above equation by explicitly diagonalizing the $Q^{(1)}$ matrix for different levels in the large $c$ limit. Note that since the central term vanishes for $L_{-1}^{\prime}$, contributions from possible $L_{-1}^{\prime m}$ to the eigenvalue are of order $1 / c$. They originate from the $s l_{2}$ (global) part of $Q^{(1)}$ and are given by

$$
\begin{aligned}
4 \hbar^{\prime} L_{-1} L_{1} L_{-1}^{\prime m}|\psi\rangle & =12 L_{-1}^{\prime} L_{1}^{\prime} L_{-1}^{\prime m}|\psi\rangle \\
& \approx \frac{12}{c}\left(m^{2}+\left(2 h_{\psi}-1\right) m\right) L_{-1}^{\prime m}|\psi\rangle,
\end{aligned}
$$

where $|\psi\rangle$ is some state of the form (27) without any $L_{-1}$ and $h_{\psi}$ is its $L_{0}$ eigenvalue.

Note that all possible off-diagonal elements in a basis built from (27) are $o(1 / c)$.

\section{Vacuum $Q$ character}

First let us only consider $Q^{(1)}$ : A suitable basis in the vacuum module $[0]$ is the set of all ordered states (27) without any $L_{-1}^{\prime}$ appearing. The vacuum $Q^{(1)}$ character is then given by

$$
\begin{aligned}
\operatorname{Tr}_{[0]} y_{1}^{Q^{(1)}} & \approx \sum_{\left\{n_{i}, k_{i}\right\} \in[\Omega]} y_{1}^{\lambda_{\left\{n_{i}, k_{i}\right\}}} \\
& =\prod_{n=2}^{\infty} \sum_{k=0}^{\infty} y_{1}^{k\left(n^{3}-n\right)}=\prod_{n=2}^{\infty} \frac{1}{1-y_{1}^{n^{3}-n}} \\
& =\prod_{n=1}^{\infty} \frac{1}{1-y_{1}^{6 \frac{n(n+1)(n+2)}{6}}} \\
\equiv 1 & +\sum_{m=1} P_{\left\{T_{n}\right\}}(m) x^{m}, \quad \text { with } \quad x=y_{1}^{6} .
\end{aligned}
$$


The numbers $T_{n}=\frac{n(n+1)(n+2)}{6}$ are the tetrahedral numbers. They are defined as the number of balls needed to construct a tetrahedral cluster with the close packing of equal spheres. From (30) one can hence show that the vacuum $Q^{(1)}$ character is a generating function for the number of partitions of an integer $m$ into tetrahedral numbers $\left\{T_{n}\right\}, P_{\left\{T_{n}\right\}}(m)$. We will see similar partitions for higher charges.

Other relevant term in $Q$ : The other nonconstant term relevant at large $c$ is $-\frac{\hbar^{\prime}(c-1)}{6} L_{0} \approx-\frac{L_{0}}{2}$. Taking it into account one obtains

$$
\operatorname{Tr}_{[0]} y_{1}^{Q} \approx \prod_{n=2}^{\infty} \sum_{k=0}^{\infty} y_{1}^{k\left(n^{3}-\frac{3}{2} n\right)}=\prod_{n=2}^{\infty} \frac{1}{1-y_{1}^{n^{3}-\frac{3}{2} n}} .
$$

The $Q^{(1)}$ eigenvalues get shifted by the (scaled) energy of the corresponding states, and at least for the low lying eigenvalues a lot of the tetrahedral degeneracy is broken. However, for large $n$, the partitions still grow exponentially in the same way as the partition into tetrahedral numbers.

\section{Primary $Q$ character}

Again first let us consider $Q^{(1)}$ : As mentioned earlier the possible $L_{-1}^{\prime}$ in the states contribute subleading to the eigenvalues with (29). For states in a primary module only containing $L_{-1}^{\prime}$ the $Q^{(1)}$ eigenvalue is even subdominant. Taking this into account we can write the $Q^{(1)}$ character in the primary $p$ module, $[p]$, as

$$
\begin{aligned}
\operatorname{Tr}_{[p]} y_{1}^{Q^{(1)}} \approx & \prod_{n=2}^{\infty} \sum_{k=0}^{\infty} y_{1}^{k\left(n^{3}-n\right)} \\
& +\left(\sum_{k=1}^{\infty} y_{1}^{\frac{12}{c}\left(k(2 p-1)+k^{2}\right)}\right) \prod_{n=2}^{\infty} \sum_{k=0}^{\infty} y_{1}^{k\left(n^{3}-n\right)} \\
\approx & \left(\sum_{k=0}^{\infty} y_{1}^{\frac{12}{c}\left(k(2 p-1)+k^{2}\right)}\right) \prod_{n=2}^{\infty} \frac{1}{1-y_{1}^{\left(n^{3}-n\right)}} .
\end{aligned}
$$

This result is the vacuum result (30) multiplied with the global character result, as given in (B20).

Full $Q$ : Again we can write the result as the vacuum character multiplied with the global character as given in (B21). It is given by

$$
\begin{aligned}
\operatorname{Tr}_{\mathrm{p}} y_{1}^{Q} & \approx\left(\sum_{k=0}^{\infty} \frac{6(p+k)^{2}+12\left(k^{2}+2 p k-k\right)}{c}-\frac{1}{2}(p+k)\right. \\
y_{1} & \prod_{n=2}^{\infty} \sum_{k=0}^{\infty} y_{1}^{\frac{1}{2} k\left(n^{3}-\frac{3}{2} n\right)} \\
& =\left(\sum_{k=0}^{\infty} y_{1}^{\frac{6(p+k)^{2}+12\left(k^{2}+2 p k-k\right)}{c}-\frac{p+k}{2}}\right) \prod_{n=2}^{\infty} \frac{1}{1-y_{1}^{\frac{1}{2}\left(n^{3}-\frac{3}{2} n\right)}} .
\end{aligned}
$$

One may estimate the term in the parentheses by extremizing the exponent with respect to $k$ and approximate the sum by the corresponding $k$ saddle value; see Sec. B 4 a.
Such an analysis, however, is expected to be quite different from the case when $c$ is fixed, since in that case the dropped terms $\propto 1 / c$ will contribute significantly to the character.

\section{B. Higher charge characters}

We now want to analyze all the higher charges in the large $c$ limit. As mentioned before, this is the classical limit which in particular means that all the quantum corrections to the classical $\mathrm{KdV}$ charges/currents can be neglected. While a closed form does not exist for the quantum charges, the classical charges are given explicitly in (A3). To compute the quantum charges in the large $c$ limit we simply have to replace $v$ in (A3) by $-2 \mathcal{L}$, multiply with $(-1)^{k}$ to obtain a positive expression, and consider the respective symmetric composite field. However, even that can be reduced further. In the classical limit we can write $\mathcal{L}=\hbar^{\prime} T-\frac{1}{8}$ (since $c \rightarrow 3 / \hbar^{\prime}$ for $\hbar^{\prime} \rightarrow 0$ ) and the $k$ th current $\left(\equiv \mathcal{I}_{k}\right)$ can be written in a power series in $\hbar^{\prime}$,

$$
(-1)^{k} \hbar^{\prime} \mathcal{I}_{k} \approx \sum_{n=0}^{k} P_{k}^{(n)}\left(T, D^{2} T, \ldots\right) \hbar^{\prime n},
$$

where $P_{k}^{(n)}\left(T, D^{2} T, \ldots\right)$ is some polynomial of grade $2 n$ of $T$ and its derivatives. $T$ contributes two and any derivative one to the grade. It follows that $P_{k}^{(n)}$ and $P_{k}^{(n+1)}$ can at most give contributions to the eigenvalues of order $c^{n / 2}$, where the eigenvectors are still given by (27). We can therefore neglect all terms in (35) with $n>2$ since they contribute at least of order $c^{-1 / 2}$ to $\mathcal{I}_{k}$. Therefore, the $k$ th current $(k>1)$ can be approximated by

$$
(-1)^{k} \hbar^{\prime} \mathcal{I}_{k} \approx \frac{C t(k-1)}{2^{2 k+1}}+P_{k}^{(1)}(T) \hbar^{\prime}+P_{k}^{(2)}(T, D T, \ldots) \hbar^{\prime 2} .
$$

$C t(k)$ is the $k$ th Catalan number and follows from the recursive definition of the classical charge. $P_{k}^{(1)}(T)$ has the form

$P_{k}^{(1)}=\frac{-\sqrt{\pi}}{(k-1) !\left|\Gamma\left(\frac{3}{2}-k\right)\right|} T(z)+$ total derivatives

and therefore contributes

$$
\frac{-\sqrt{\pi}}{(k-1) !\left|\Gamma\left(\frac{3}{2}-k\right)\right|} L_{0}
$$

to the charge.

The remaining polynomial can be written as

$$
P_{k}^{(2)}=\sum_{m=0}^{k-2} b_{m}^{k} T(z) D^{2 m} T(z) \quad \text { for } k \geq 2
$$


with $b_{m}^{k}$ positive constants, where always $b_{k-2}^{k}=2$. The other coefficients can e.g., be computed by the help of a computer algebra program. Integrating over this part of the current then gives

$\frac{1}{2 \pi i \hbar^{\prime}} \oint \frac{d z}{z} P_{k}^{(2)} \hbar^{\prime 2}=2 \hbar^{\prime} \sum_{n>0}\left(\sum_{m=0}^{k-2}(-1)^{m} b_{m}^{k} n^{2 m}\right) L_{-n} L_{n}$,

where for now we omit the term proportional to $L_{0}^{2}$ which is subleading in large $c$. The relevant part of the $k$ th charge in the large $c$ limit can therefore be written as

$$
\begin{aligned}
(-1)^{k} \hat{K}_{k}= & 2 \hbar^{\prime} \sum_{n>0}\left(\sum_{m=0}^{k-2}(-1)^{m} b_{m}^{k} n^{2 m}\right) L_{-n} L_{n} \\
& -\frac{\sqrt{\pi}}{(k-1) !\left|\Gamma\left(\frac{3}{2}-k\right)\right|} L_{0}+\frac{C t(k-1)}{2^{2 k+1} \hbar^{\prime}}, \\
\equiv & K_{k}^{(1)}-\frac{\sqrt{\pi}}{(k-1) !\left|\Gamma\left(\frac{3}{2}-k\right)\right|} L_{0}+\frac{C t(k-1)}{2^{2 k+1} \hbar^{\prime}} .
\end{aligned}
$$

With this it is clear that the previously chosen orthogonal basis in large $c$ diagonalizes all the higher charges as well. First, note that we have kept only the leading order terms in large $c$. At $\mathcal{O}(1 / c)$, the only contributions come from the $L_{0}^{2}$ and the $L_{-1} L_{1}$ term in $K_{k}^{(1)}$. Second, note that the most suppressed term is

$$
(-1)^{k} \hbar^{k-1} 2^{k-1} C t(k-1) L_{0}^{k} \approx(-1)^{k} \frac{6^{k-1}}{c^{k-1}} C t(k-1) L_{0}^{k} .
$$

\section{1. $\hat{K}_{k}$ vacuum character}

Now, we focus on the vacuum module. Using (41) we obtain

$$
\hat{K}_{k}\left|\left\{n_{i}, l_{i}\right\}\right\rangle=\mathfrak{\Re}_{k,\left\{n_{i}, l_{i}\right\}}\left|\left\{n_{i}, l_{i}\right\}\right\rangle
$$

with

$$
\begin{aligned}
\mathfrak{\Re}_{k,\left\{n_{i}, l_{i}\right\}} & =\sum_{i=1}^{M} l_{i}\left[\left(n_{i}^{3}-n_{i}\right)\left(\sum_{m=0}^{k-2}(-1)^{k+m} \frac{b_{m}^{k}}{2} n_{i}^{2 m}\right)-\frac{(-1)^{k} \sqrt{\pi} n_{i}}{(k-1) !\left|\Gamma\left(\frac{3}{2}-k\right)\right|}\right]+\frac{(-1)^{k} C t(k-1)}{2^{2 k+1} \hbar^{\prime}} \\
& \equiv \sum_{i=1}^{M} l_{i}\left[\mathfrak{p}_{k, n_{i}}^{(1)}-\frac{(-1)^{k} \sqrt{\pi} n_{i}}{(k-1) !\left|\Gamma\left(\frac{3}{2}-k\right)\right|}\right]+(-1)^{k} \frac{C t(k-1)}{2^{2 k+1} \hbar^{\prime}}
\end{aligned}
$$

Focusing on the $K^{(1)}$ part of the $l$ th $\mathrm{KdV}$ charge, we find the character analogous to (30) as

$$
\operatorname{Tr}_{[0]} y_{l}^{K_{l}^{(1)}} \approx \prod_{n=2}^{\infty} \sum_{k=0}^{\infty} y_{l}^{k \cdot \mathfrak{p}_{l, n}^{(1)}}=\prod_{n=2}^{\infty} \frac{1}{1-y_{l}^{\mathfrak{p}_{l, n}^{(1)}}}
$$

For $\mu_{l} \ll 1\left(\mu_{l}=-\log y_{l}\right)$ we can assume that the above is dominated by large $n \sim n_{*} \gg 1 .^{5}$ For large enough $n$ we can approximate $\mathfrak{p}_{l, n_{i}}^{(1)} \approx n^{2 l-1}$ and, hence, obtain

$$
\operatorname{Tr}_{[0]} y_{l}^{K_{l}^{(1)}} \approx \prod_{n=2}^{\infty}\left(1-y_{l}^{n^{2 l-1}}\right)^{-1} \text {. }
$$

Remarkably, Eq. (46) can be expressed in an expansion in $y_{l}$ weighted by power partitions, $p_{l}\left(q_{m}\right)$,

$$
\operatorname{Tr}_{[0]} y_{l}^{K_{l}^{(1)}} \approx \sum_{q_{m}=0}^{\infty} p_{l}\left(q_{m}\right) y_{l}^{q_{m}} .
$$

\footnotetext{
${ }^{5}$ However, since in our approximation $c \gg n_{*}$, we will also obtain a lower bound on $\mu_{l}$.
}

$p_{l}\left(q_{m}\right)$ is the number of ways $q_{m}$ can be expressed as the sum of integers which are of the form $i^{2 l-1}$ where $i$ is also an integer (this is the quantum number of the $l$ th $\mathrm{KdV}$ charge). Note that for $l=2$, this is the partition of $n$ into cubes, which is the large $n$ asymptotics of tetrahedral numbers. It turns out that there is a formula due to Hardy and Ramanujan [[21] p. 111] which gives an asymptotic analytic form for the power partitions,

$$
p_{l}\left(q_{m}\right) \approx \exp \left(A(l) q_{m}^{\frac{1}{2}}\right)
$$

where $A(l)=2 l\left(\frac{1}{2 l-1} \Gamma\left(\frac{2 l}{2 l-1}\right) \zeta\left(\frac{2 l}{2 l-1}\right)\right)^{(2 l-1) /(2 l)}$. This then gives us the density of states in the vacuum module with $K_{l}^{(1)}$ charge $q_{m}$, where $q_{m} \gg 1$. Adding the $L_{0}$ contribution does not change the above logic which allows us to approximate the vacuum character by

$$
\operatorname{Tr}_{[0]} y_{l}^{K_{l}} \approx \sum_{q_{m}=0}^{\infty} \exp \left(A(l) q_{m}^{\frac{1}{2 l}}-\mu_{l}\left(q_{m}-c B_{l}\right)\right)
$$


where $B_{l}=(-1)^{l+1} \frac{C t(l-1)}{32^{2 l+1}}$ comes from the constant part in the charge. We can further approximate the sum by an integral and obtain

$$
\begin{aligned}
\operatorname{Tr}_{[0]} y_{l}^{K_{l}} & \approx e^{\mu_{l} c B_{l}} \int_{0}^{\infty} d q_{n} \exp \left(A(l) q_{n}^{\frac{1}{2 l}}-\mu_{l} q_{n}\right) \\
& \approx e^{\mu_{l} c B_{l}} \exp \left\{\frac{2 l-1}{2 l} A(l)^{\frac{2 l}{2 l-1}}\left(2 l \mu_{l}\right)^{\frac{1}{(1-2 l)}}\right\},
\end{aligned}
$$

where the integral has been done by saddle in (C3).

\section{2. $\hat{K}_{k}$ primary characters}

Only $K_{l}^{(1)}$ : As in the case of $Q^{(1)}$ we only have to consider the additional contributions from $L_{-1} L_{1}$ in the sum. It is subleading and adds

$$
\frac{24}{c}\left(k(2(p+N)-1)+k^{2}\right) \mathfrak{A}
$$

to the dominant contribution, where $N$ denotes the contribution to the conformal dimension from all excitations not including $L_{-1}$ and $\mathfrak{A}=\sum_{m=0}^{l-2}(-1)^{m} b_{m}^{l}$. Analogously to $Q^{(1)}$ we can therefore write

$$
\begin{aligned}
\operatorname{Tr}_{[p]} y_{l}^{K_{l}^{(1)} \approx} & \prod_{n=2}^{\infty} \sum_{k=0}^{\infty} y_{l}^{k \mathfrak{p}_{l, n}^{(1)}} \\
& +\left(\sum_{k=1}^{\infty} y_{1}^{\frac{12}{c}\left(k(2 p-1)+k^{2}\right) \mathfrak{A}}\right) \prod_{n=2}^{\infty} \sum_{k=0}^{\infty} y_{l}^{k\left(\mathfrak{p}_{l, n}^{(1)}+\frac{24}{c} \mathfrak{A} n\right)} \\
\approx & \left(\sum_{k=0}^{\infty} y_{1}^{\frac{12}{c} \mathfrak{A}\left(k(2 p-1)+k^{2}\right)}\right) \prod_{n=2}^{\infty} \frac{1}{1-y_{l}^{\mathfrak{p}_{l, n}^{(1)}}}
\end{aligned}
$$

Again, this is the global answer multiplied with the vacuum result.

Full $K_{l}$ : Again, analogous to the case of $Q$, the dominant contributions from $L_{-1}$ to states in the $p$-module come from the part in $\hat{K}_{l}$ proportional to $L_{0}$. Formally, we can write

$$
\begin{aligned}
\operatorname{Tr}_{[p]} y_{l}^{K_{l}} \approx & \left(\sum_{k=0}^{\infty} \frac{-\sqrt{\pi}}{y_{1}^{\left.(k-1) ! ! \Gamma \frac{3}{2}-k\right) !}(p+k)+\frac{12}{c}\left(k(2 p-1)+k^{2}\right) \mathscr{A}+\frac{b_{0}^{l}}{c}(p+k)^{2}}\right) \\
& \times \prod_{n=2}^{\infty} \frac{1}{1-y_{l}} \frac{\mathfrak{p}_{k, n}^{(1)}+\frac{-\sqrt{\pi} n}{(k-1) ! \Gamma\left(\frac{3}{2}-k\right) \mid}}{}
\end{aligned}
$$

\section{Comment on the full GGE}

The full generalized Gibbs ensemble is obtained by taking the sum over all GGE characters that appear in the CFT. This sum, however, depends on the specifics of the theory. Note that we cannot approximate the result by e.g., taking the asymptotic formula for the growth of primary states $[8,9]$, because this formula is valid in energy regimes $\left(\Delta_{p} \gg c\right)$ that do not fit the regimes of validity that we consider $\left(\Delta_{p} \ll c\right)$. We would need the specifics of the spectrum at intermediate energies which is not universal [22].

\section{Constraints on $\boldsymbol{\mu}_{l}$}

We already mentioned it earlier and now want to show that one gets a lower bound on $\mu_{l}$ by demanding that the dominant contribution from the saddle point of the above integral is smaller than $c$. The saddle turns out to be

$$
\begin{aligned}
& 1 \ll q_{n}^{*}=2^{\frac{2 l}{1-2 l}}\left(\frac{l \mu_{l}}{A(l)}\right)^{\frac{2 l}{1-2 l}} \ll c \\
\Rightarrow \mu_{l} & \gg \frac{A(l)}{2 l} \frac{1}{c^{1-\frac{1}{2 l}}} \approx \frac{1}{c^{1-\frac{1}{2 l}}} .
\end{aligned}
$$

This implies that our result (51) is valid in the regime

$$
c^{\frac{1}{2 l}-1} \ll \mu_{l} \lll 1 .
$$

\section{CONNECTION BETWEEN HIGH AND LOW CHEMICAL POTENTIALS}

As mentioned in our Introduction one of our motivations to study integrable charges and the GGE is to reveal some possible transformation property of the chemical potential under which the full GGE or GGE characters behave in a certain way. In this section we show that our results allow one to discuss some aspects of the transformation.

First, let us collect the results at large and small chemical potentials and large $c$ with a single integrable charge $\hat{K}_{l}$. At a high chemical potential the corresponding GGE is dominated by the constant part of the charge because it has the highest power in $c$. However, the contribution is not necessarily given by the vacuum contribution because the charges are not necessarily positive. The state with the lowest charge eigenvalue dominates. Nevertheless we can only restrict ourselves to the constant part, because all other contributions in this regime are hugely subdominant. So at large chemical potential $1 \ll \mu_{l} \ll c$, and with all other chemical potentials turned off, we can approximate the GGE by

$$
Z\left(\mu_{l}\right) \approx e^{\mu_{l} c B_{l}},
$$

where $B_{l}=(-1)^{l+1} \frac{C t(l-1)}{2^{2 l+1}}$ is the constant part computed in the last section.

In the regime of small chemical potential, $1 / c^{1-1 / 2 l} \ll$ $\mu_{l} \ll 1$, we in particular computed the $\hat{K}_{l}$ vacuum character in (51), 


$$
\operatorname{Tr}_{[0]} y_{l}^{\hat{K}_{l}} \approx e^{\mu_{l} c B_{l}} \exp \left\{\frac{\mathcal{C}_{l}}{\frac{1}{\mu_{l}^{2-1}}}\right\},
$$

where $\mathcal{C}_{l}=\Gamma\left(\frac{2 l}{2 l-1}\right) \zeta\left(\frac{2 l}{2 l-1}\right)$. The factor in the front, $e^{\mu_{l} c B_{l}}$, again comes from the constant part. However, in the case of small chemical potentials we can show that this factor is negligible for large $l$. The competition is between the factors $\mu_{l} c B_{l}$ and $\mathcal{C}_{l} \mu_{l}^{\frac{1}{1-2 l}}$. When $\mu_{l}$ is taken as the smallest it can be (to be in the valid regime of our analysis, i.e., $\mu_{l} \sim c^{\left.-1+\frac{1}{2 l}\right)}$, the two terms are of the order $\sqrt{c^{l}} B_{l}$ and $\sqrt{c^{l}} \mathcal{C}_{l}$. Therefore we need to compare $\mathcal{C}_{l}$ and $B_{l}$. For large $l$, using the asymptotics of Gamma and Zeta functions, $\mathcal{C}_{l} \sim 2 l-1$, whereas using the asymptotics of Catalan numbers $\left|B_{l}\right| \sim l^{-3 / 2} /(8 \sqrt{\pi})$. Therefore clearly, for small $\mu_{l}$, as $l$ gets bigger,

$$
\operatorname{Tr}_{[0]} y_{l}^{\hat{K}_{l}} \approx \exp \left\{\frac{\mathcal{C}_{l}}{\mu_{l}^{\frac{1}{2 l-1}}}\right\} .
$$

If we now assume that there exist any nice transformation property that relates the low and high chemical potentials relating the respective GGEs, it is reflected in the second factor $\exp \left\{\mathcal{C}_{l} / \mu_{l}^{\frac{1}{(2 l-1)}}\right\}$. Since the vacuum character appears in all primary characters, we can assume that this factor multiplies the full GGE.

It follows immediately that, if $\mu_{i} \neq \beta$, it is not possible to connect the low and high potential regime by an " $S$ transformation," $\mu_{i} \rightsquigarrow \frac{1}{\mu_{i}}$. Rather, the transformation that connects the high and low chemical potential regimes seems to have the form

$$
\mu_{i} \rightarrow \mathcal{D}_{i} \mu_{i}^{\frac{1}{1-2 i}}
$$

where $\mathcal{D}_{i}$ is independent of $\mu_{i}$, just depending on the charge and $c$. It is clear that the GGE can definitely not be invariant under this transformation. This is because any function of $\mu_{i}$ that is invariant under such a transformation would be constant. This follows easily by performing $\mu_{i} \rightarrow \mathcal{D}_{i} \mu_{i}^{\frac{1}{1-2 i}}$ several times,

$f\left(\mu_{i}\right)=f\left(\mathcal{D}_{i}^{\sum_{j=0(1-2 i)^{j}}^{k-1}} \mu_{i}^{\frac{1}{(1-2 i)^{k}}}\right) \quad \forall k \in \mathbb{N}, \mu_{i} \in \mathbb{R}^{+}$.

Taking the limit $k \rightarrow \infty$ then tells that $f\left(\mu_{i}\right)=f\left(\mathcal{D}_{i}^{\frac{2 i-1}{2 i}}\right)$.

\section{A. Connection to very high energies}

Recent study of $\mathrm{KdV}$ in 2D CFTs have explored various aspects of the GGE in the regimes of asymptotically large energies and $\mathrm{KdV}$ charges [14-16]. In particular, the chemical potentials are outside the regime that we have studied, viz., $\mu_{i} \ll \frac{1}{c^{1-1 / 2 i}}$. Therefore in this regime, the GGE partition function is dominated by $h_{*}, n_{*} \gg c$. Additionally, the investigations also focus on the limit, $\left\{\mu_{l}\right\} \ll \beta \ll 1$, therefore the $\mathrm{KdV}$ charges are perturbatively treated on the torus. It was shown that when high energies dominate, the main contribution to the GGE comes from descendants at a particular level of primaries at a particular energy regime, and the GGE can be approximated by [using (42)] ${ }^{6}$

$$
\begin{aligned}
& Z\left(\left\{q, y_{i}\right\}\right) \\
& \quad=\operatorname{Tr} q^{H} \prod_{i} y_{i}^{\hat{K}_{i}} \\
& \quad \approx \int_{0}^{\infty} d h \rho(h) \exp \left(-\beta h-\sum_{i} \mu_{i} \frac{6^{i-1} C t(i-1)}{c^{i-1}} h^{i}\right) .
\end{aligned}
$$

In this high energy regime unlike [14-16], we shall focus on $1 \gg\left\{\mu_{l}\right\} \gg \beta$. The integral can in principle be solved by a saddle point approximation. This, however, becomes

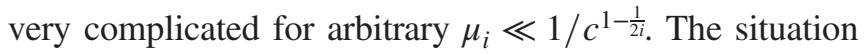
simplifies when we take all potentials parametrically small with respect to one particular potential. In that case, the saddle is always dominated by the contribution of that particular potential with corrections given by a power series in all the other ones. The dominant saddle $h^{*}$ for $\mu_{n} \gg\left\{\mu_{i}\right\}, \beta$ is given by

$h^{*}=\left(\frac{\pi}{n 6^{i-1 / 2} C t(i-1) \mu_{n}}\right)^{\frac{2}{2 n-1}} c+\sum_{i \neq n} C_{i} \mu_{i}+\mathcal{O}\left(\mu_{i} \mu_{j}\right)$,

where the coefficients $C_{i} \equiv C_{i}\left(\mu_{n}, c\right)$ are such that the corrections are small. Plugging back this saddle, the partition function is approximately given by

$$
\begin{aligned}
& Z(1\left.\gg \mu_{n} \gg \mu_{i}\right) \\
& \quad \approx \exp \left[\frac{(2 n-1) c}{6}\left(\frac{\pi^{2 n}}{C t(n-1) n^{2 n} \mu_{n}}\right)^{\frac{1}{2 n-1}}+\mathcal{O}\left(\mu_{i}\right)\right] .
\end{aligned}
$$

Thus a bit surprisingly, we see that the GGE for very low chemical potential $\mu_{l} \ll \frac{1}{c^{1-1 / 2 l}}$ shows the same $\mu_{l}$ dependence that we saw when we analyzed the universal vacuum character in the range $\frac{1}{c^{1-1 / 2 l}} \ll \mu_{l} \ll 1$, (57).

This is also compatible with the known results from the thermal partition function. The Virasoro characters and the

\footnotetext{
${ }^{6}$ Our coefficients in the exponent differ from those in [14-16], since the expressions for $\mathrm{KdV}$ charges (for $c>1$ ) are different. This is because we have chosen the shift to $T(z)$ to be $\frac{1-c}{24}$ as in [1] while in [3-5] the shift is $-\frac{c}{24}$, which is followed by [14-16]. However, the large $c$ expressions of course match.
} 
full partition function at high temperature show qualitatively the same behavior. The sum over all characters must lead to a quantitative agreement and, hence, gives tight constraints on the spectrum of the theory. The latter e.g., allows one to compute the asymptotic growth of the density of primary states. In the GGE case, using (62) we can derive the growth of the asymptotic density of states carrying the $n$th KdV charge $\left(q_{n}\right)$ as $\exp \left(A q_{n}^{\frac{1}{2 n}}\right)$ [same $q_{n}$ dependence as in (48)].

\section{COMMENT ON APPROXIMATIONS OF PURE STATES}

Studying the GGE can also shed more light on how the ETH [23-25] might be more generally realized in twodimensional conformal field theory. It states that for a particular class of simple operators generic energy eigenstates can be well approximated by thermal states. It is, however, only expected to be true in theories exhibiting chaotic dynamics and known to fail in integrable theories, where integrable here not only means that there exists an infinite set of conserved charges but also that these trivialize the dynamics. In case of 2D CFTs with $c>1$ we saw that there exist an infinite set of conserved charges. However, the dynamics is not necessarily trivial. For example in [26] it has been shown that 2D CFTs exhibit chaotic dynamics and, hence, a generalization of ETH might hold. The generalization takes not only the Hamiltonian/energy into account but considers all conserved quantities. One might expect that a generic static state can for some class of simple operators be approximated by some equilibrium state. The latter defines a GGE by taking the trace over the spectrum of the theory.

We, here, want to check how well a GGE dominated by a single chemical potential, $\mu_{l}$, can approximate a typical eigenstate of the corresponding $\mathrm{KdV}$ charge, $\hat{K}_{l}$, in the regime that we probe. For now let us choose such a state $\left|q_{m}\right\rangle$, whose $\hat{K}_{l}$ eigenvalue is $q_{m}-c B_{l}$, where $1 \ll q_{m} \ll c$. Restricting to the vacuum part of the GGE, in the regime $\left\{\mu_{i}, \beta\right\} \ll \mu_{l} \ll 1$, then we can compute $\left\langle\hat{K}_{l}\right\rangle$ in the mixed state $e^{-\mu_{l} \hat{K}_{l}}$ reduced to the vacuum module with the help of (51) as

$$
\begin{aligned}
\left\langle\hat{K}_{l}\right\rangle_{q_{l}} & =-\partial_{\mu_{l}} \log Z_{\mathrm{GGE}}^{0, l} \\
& \approx-\partial_{\mu_{l}}\left\{\frac{2 l-1}{2 l} A(l)^{\frac{2 l}{2 l-1}}\left(2 l \mu_{l}\right)^{\frac{1}{1-2 l}}+\mu_{l} c B_{l}\right\} \\
& =\left(2 l A(l) \mu_{l}\right)^{\frac{2 l}{1-2 l}}-c B_{l} .
\end{aligned}
$$

Hence, a necessary condition for $e^{-\mu_{l} \hat{K}_{l}}$ being a good approximation to $\left|q_{m}\right\rangle\left\langle q_{m}\right|$ is when we equalize the eigenvalue, $q_{m}-c B_{l}$ to the above ensemble answer. This gives

$$
\mu_{l}=\frac{1}{2 l A(l)} q_{m}^{\frac{1-2 l}{2 l}}
$$

Note that since the GGE should be a positive quantity, $\mu_{l}$ should be a real quantity, implying positivity of $q_{m}$. Thus in the cases where $q_{m}<0$ it appears that we cannot have equilibration. In the regime we consider, this can e.g., happen if the state happens to have zero excitations or a lot of light excitations viz. $L_{-1}^{k}$. Then the term proportional to $\sum_{n>1} L_{-n} L_{n}$ contributes not enough to the charge to overcome the negative contribution from the term proportional to $L_{0}$. This also is an artifact of the large $c$ regime we are working in, since higher powers of $L_{0}$ [which are subleading in $\mathcal{O}(1 / c)$ ] can make $q_{m}>0$ when one goes beyond our regime and in particular to much higher energies.

It is interesting to note that states close to the primary, in the sense that there are almost no excitations on top of it, can be well approximated by a purely thermal state in the large $c$ limit [27]. However, in [27] they also conjectureand show it for particular states explicitly-that descendants far from their primary states cannot be approximated well by a purely thermal state, even at large $c$. For such states, $q_{m} \gg 0$ and GGE states might be a necessity for a good approximation of those.

\section{CONCLUSIONS}

With this note we have shed some light on the implications that follow from the integrable structure of 2D CFT. We have first shown under which conditions one not only can construct an infinite set of commuting charges in a CFT with $c>1$ but also can connect it to the quantum sinhGordon theory. Our analysis implies that if one can write the energy momentum tensor in the Feigin-Fuchs form, then the quantum KdV charges of CFT with $c=13+\frac{3}{\hbar^{\prime}}+$ $12 \hbar \geq 25$ are related to the quantum $\mathrm{mKdV}$ charges of sinh-Gordon (sG) theory with the Hamiltonian

$$
\hat{H}=\frac{1}{2 \pi i} \oint \frac{d z}{z}\left(1-\frac{1}{2 z^{\frac{h^{\prime}}{2}}}\left(: e^{\beta \phi}:+: e^{-\beta \phi}:\right)\right) .
$$

The relation between the quantum $\mathrm{KdV}$ and quantum $\mathrm{mKdV}$ charges is given by the Miura transform (18).

Next we have concentrated on the KdV characters at a large central charge which on the sG side corresponds to taking the classical limit $(\hbar \sim 3 / c \rightarrow 0)$. They are the building blocks of generalized Gibbs ensembles and can help in understanding its general features. The $\mathrm{KdV}$ characters can be computed for any integrable charge $\hat{K}_{l}$, as long as the corresponding chemical potential $\mu_{l}$ is bounded from below by $1 / c^{1-\frac{1}{2 l}}$. This probes regimes where the corresponding eigenvalues of the charge are small compared to the central charge, which is always kept parametrically large in our analysis. The key idea is that to 
leading order in large $c$ all states of the form (27) diagonalize the charges with eigenvalues given by (44).

A key motivation behind this work has been whether there exists a connection between the high and low chemical potential GGEs. By using the HardyRamanujan result (48) on the number of partitions into monomials we can find the behavior of the KdV vacuum character at small chemical potential (51). This factor appears in the other characters as well and is universal. The self-consistency of our analysis bounds the chemical potential $\mu_{l}$ from below by $1 / c^{1-\frac{1}{2 l}}$. When considering much lower chemical potentials, s.t. eigenvalues much larger than $c$ dominate the GGE, one can also extract the result for the full GGE (62) from the results in [14]. Both methods show the same $\mu_{l}$ dependence in the GGE answer, which suggests that this behavior is valid for a rather large range of $\mu_{l} \ll 1$. On the other end, for large $\mu_{l}$, the GGE is dominated by the vacuum. In the case of the first KdV charge, namely the Hamiltonian, the two regimes are directly related by the standard $\mathcal{S}$-transformation, which takes $\beta \rightarrow 1 / \beta$. It turns out that for the higher chemical potentials a transformation of the form, $\mu_{l} \rightarrow \mu_{l}^{\frac{1}{1-2 l}}$, Eq. (58) relates the low and the high $\mu_{l}$ GGEs.

We also briefly commented on a generalization of the eigenstate thermalization hypothesis. If a thermal state fails to approximate an energy eigenstate, it might be cured by considering GGE states. Once again in the restricted regime of a single chemical potential dominated GGE, we show the conditions when such an approximation is well defined. States that are already known to be approximated well by a thermal state, namely high energy primary states and descendant states with only light excitations on top of the primary [27], can —at least in the large $c$ regime of validity of our analysis - not be approximated by a GGE state with only a single charge other than the Hamiltonian turned on, since the corresponding chemical potential is then no longer real. On the other hand, for pure states made out of large excitations above the highest weight state, one can solve for the GGE chemical potential that needs to be turned on in (64).

A number of interesting directions are left for future work. Here we list some of the more pressing ones:

(i) Going beyond the large $c$ approximation: to proceed in the $1 / c$ calculation of the GGE character, one needs to know more subleading terms in $K_{k}$ beyond the regime of the present analysis. In future work, we shall try to see if the issue of finite $c$ can be addressed by extending the technologies developed in [3-5] to $c>1$. Since the problem of finding the eigenvalues of all $\mathrm{KdV}$ charges in the CFT can be mapped to the problem of solving a particle in a specific potential, it might also be possible that symmetries of the GGE are directly related to symmetries in the spectrum of the quantum particle. (ii) Investigate the GGE in specific models: until now, the GGE with only the second charge turned on has not even been computed for free theories. We in particular hope that a closed form of the GGE might reveal more general transformation properties of the chemical potentials and show that the connection between the high and low chemical potentials that we see in this paper is not an artifact of taking large $c$. For free bosons, the largest eigenvalue of $K_{2}$ at level $n$ seems to grow as $n^{3}$ [28].

(iii) Issues of equilibration: the pure states in 2D CFT are of the form $|\psi\rangle=\prod_{i} L_{-n_{i}}^{k_{i}}|p\rangle$, where $|p\rangle$ is a highest weight state, with $L_{0}$ eigenvalue $E=p+\sum_{i} n_{i} k_{i}$. Hence the thermal ensemble is only sensitive to the number $E$. On the other hand, to fix a KdV GGE one has to use more information regarding the construction of the state. There are clear indications of this even in large $c$. For example, the large $c$, eigenvalue $\lambda_{\left\{n_{i}, k_{i}\right\}}$ of the second KdV charge, Eq. (28), is not just the combination $E$, and thus probes a finer microstructure of $|\psi\rangle$. Higher KdV charges probe even finer microstructures; however, to understand this completely, one will need to go beyond large $c$, since in leading order in $1 / c$, the $\lambda_{\left\{n_{i}, k_{i}\right\}}$ eigenvalues continue to dominate.

(iv) Holographic interpretation: adding polynomials of the stress tensor and its derivatives with respective chemical potentials to the action of a CFT corresponds to a multitrace deformation in the CFT. In a potential dual description of the CFT these deformations also appear as multitrace deformation of the action of gravitational action (or its higher spin extension). As discussed in [29] these can for example be studied in the Chern-Simons formulation. The deformation appears as a boundary term which does not change the bulk field equations, and hence a classical BTZ black hole solution stays unchanged. However, the free energy/partition function is different from that of the usual BTZ black hole because of the additional boundary terms. In fact, the change looks like a generalized Legendre transform which changes the partition function to the so-called tau-function of the classical $\mathrm{KdV}$ hierarchy. When quantizing the Chern-Simons theory with matter this leads to more structure in the gravitational solution and could lead to explicit constructions of black holes with quantum hair.

Apart from the above, it will also be interesting to understand the geometric interpretation of the $\mathrm{KdV}$ charges, in the same way that the Hamiltonian translates states along the Euclidean time. A generalization of the mapping of GGE to a quantum mechanics problem [3-5] for $c>1$ and for arbitrary chemical potentials is another fascinating future direction. 


\section{ATTACHMENTS}

We uploaded two Mathmatica ${ }^{\circledR}$ notebooks which can be found from http://home.iitk.ac.in/ didas/kdv/1901notebooks .html. The file LARGEC.NB is concerned with explicit checks of the large $c$ eigenvalues of the second charge. This notebook uses VIRASORO.NB by Matthew Headrick, which can be downloaded from http://people.brandeis.edu/headrick/ Mathematica/Virasoro.nb. Finally in KDV_CHARGES.NB we explicitly construct the leading order parts of the first $n$ charges in large $c$. It in particular confirms the coefficients we give in Sec. III B.

\section{ACKNOWLEDGMENTS}

It is a pleasure to thank George Jorjadze and Stefan Theisen for encouragement and useful discussions at various stages of the project. We also thank Shouvik Datta who was part of the initial collaboration. D. D. is grateful to the MPI Partner group Grant No. MAXPLA/ PHY/2018577.

\section{APPENDIX A: CLASSICAL INTEGRALS OF MOTION}

\section{Integral of motions for the modified $\mathrm{KdV}$ equation}

The classical integrals of motion defining the $\mathrm{mKdV}$ hierarchy are given by

$$
I_{n}=-\frac{1}{2 \pi} \int_{0}^{2 \pi} d \sigma \frac{1}{2} u(\sigma) Y_{2 n-1}(u)
$$

with

$$
Y_{n+1}=\partial_{\sigma} Y_{n}+u \sum_{k=1}^{n-1} Y_{k} Y_{n-k}, \quad Y_{1}=-u
$$

\section{Integral of motions for the $\mathrm{KdV}$ equation}

The classical integrals of motion defining the $\mathrm{KdV}$ hierarchy are given by

$$
K_{n}(v)=\frac{1}{2 \pi} \int_{0}^{2 \pi} d \sigma \frac{1}{2} v(\sigma) Z_{2 n+1}(v)
$$

with

$$
Z_{n+1}=\partial_{\sigma} Z_{n}+v \sum_{k=1}^{n-1} Z_{k} Z_{n-k}, \quad Z_{1}=1
$$

\section{APPENDIX B: QUANTUM KDV CHARGE OF 2D CFTs}

\section{Composite operators}

A composite operator $A B(z)$ of two quantum fields $A(z)=\sum_{n} A_{n} z^{-n}$ and $B(z)=\sum_{n} B_{n} z^{-n}$ is defined as

$$
A B(z) \equiv \frac{1}{2 \pi i} \oint_{\mathcal{C}_{z}} \frac{d \zeta}{\zeta} \frac{z}{\zeta-z} \mathcal{R}(A(\zeta) B(z))
$$

where $\mathcal{R}$ denotes radial ordering, i.e.,

$$
\mathcal{R}(A(\zeta) B(z))= \begin{cases}A(\zeta) B(z) & \text { for }|\zeta| \geq|z| \\ B(z) A(\zeta) & \text { for }|\zeta|<|z|\end{cases}
$$

The modes of the composite field are then given by

$$
A B_{n}=\sum_{l=1}^{\infty} A_{-l} B_{l+n}+\sum_{l=0}^{\infty} B_{n-l} A_{l}
$$

This definition gives you some sort of normal ordering with respect to the modes $A_{n}$ and $B_{n}$. However, note that there is no additional normal ordering part. The composition of several operators is always defined by first composing the leftmost operators and successively composing the operators that follow to the right; e.g., $A B C(z)$ is defined by composing $A B(z)$ with $C(z)$.

Furthermore, any composite operator from now on is understood as a symmetric composition, i.e.,

$$
\begin{aligned}
\langle A B\rangle & =\frac{1}{2}(A B(z)+B A(z)), \\
\left\langle A_{1} A_{2} \ldots A_{n}\right\rangle & =\frac{1}{n !} \sum_{p} A_{p_{1}} A_{p_{2}} \cdots A_{p_{n}},
\end{aligned}
$$

where the sum is taken over all permutations and we omit the brackets in what follows. 


\section{The charges}

The first few charges are given by (see also [1])

$$
\begin{aligned}
H \equiv & \hat{K}_{1}=\frac{1}{2 \pi \hbar^{\prime} i} \oint \frac{d z}{z} \mathcal{L}(z)=L_{0}-\frac{c-1}{24}, \\
Q \equiv & \hat{K}_{2}=\frac{1}{2 \pi \hbar^{\prime} i} \oint \frac{d z}{z} 2 \mathcal{L}^{2}(z)=2 \hbar^{\prime}\left(2 \sum_{n=1}^{\infty} L_{-n} L_{n}+L_{0}^{2}-\frac{c-1}{12} L_{0}+\frac{(1-c)^{2}}{576}\right) \\
\hat{K}_{3}= & \frac{1}{2 \pi \hbar^{\prime} i} \oint \frac{d z}{z} 8 \mathcal{L}^{3}+\frac{2 \hbar^{\prime}}{3}(c+2)(D \mathcal{L})^{2} \\
= & 8 \hbar^{\prime 2}\left(\sum_{n, m=1}^{\infty} 2 L_{-m} L_{-n+m} L_{n}+L_{-n-m} L_{m} L_{n}+L_{-n} L_{-m} L_{n+m}+3 \sum_{n=1}^{\infty} L_{-n} L_{0} L_{n}-\frac{2(c+2) n^{2}-36 n+3(c-1)}{12} L_{-n} L_{n}\right. \\
& \left.+L_{0}^{3}+\frac{1-c}{8} L_{0}^{2}+\frac{(1-c)^{2}}{192} L_{0}+\frac{(1-c)^{3}}{13824}\right), \\
K_{4}= & \frac{40}{2 \pi \hbar^{\prime} i} \oint \frac{d z}{z}\left[\mathcal{L}^{4}+\frac{\hbar^{\prime}}{3}(c+2) \mathcal{L}(D \mathcal{L})^{2}+\frac{\hbar^{\prime 2}}{180}(c+2)\left(c-\frac{1}{2}\right)\left(D^{2} \mathcal{L}\right)^{2}-\frac{\hbar^{\prime 2}}{24}(c+2)(D \mathcal{L})^{2}\right] .
\end{aligned}
$$

In the limit $\hbar^{\prime} \rightarrow \frac{3}{c} \rightarrow 0$ they converge to the classical $\mathrm{KdV}$ charges (A3) when we replace $\mathcal{L}=\frac{v}{2}$ up to an overall factor of $\frac{1}{\hbar^{\prime}}$.

\section{Some explicit checks on large $c$ eigenvalues of $Q$}

We can in principle compute the eigenvalues of $\tilde{Q}^{(1)}=$ $2 \sum_{n=1}^{\infty} L_{-n} L_{n}$ with the help of Mathematica up to arbitrary level. However, the calculation time grows rapidly with the level. We here give the results up to level 6 in the vacuum module and show that the large $c$ eigenvalues are given by $k_{i} \frac{n_{i}^{3}-n_{i}}{6} c$.

Levels 0 and 1: $\tilde{Q}^{(1)}$ annihilates the vacuum and there is no level 1 in the vacuum module. So no contribution here.

Level 2: A (not normalized) basis is $\left\{L_{-2}|0\rangle\right\}$. Its only basis element's eigenvalue is simply $c$ and matches $c \frac{n^{3}-n}{6} \stackrel{n=2}{=} c$.

Level 3: A (not normalized) basis is $\left\{L_{-3}|0\rangle\right\}$. Its only basis element's eigenvalue is $4 c+4$. In the large $c$ limit this gives $4 c=\frac{3^{3}-3}{6} c$.

Level 4: A (not orthonormal) basis is $\left\{L_{-4}|0\rangle, L_{-2}^{2}|0\rangle\right\}$. The matrix representation of $\tilde{Q}^{(1)}$ in this basis is

$$
\left(\begin{array}{cc}
10 c+20 & 3 c+6 \\
6 & c+8
\end{array}\right)
$$

with eigenvalues $\lambda_{1 / 2}=9+3 c \pm \sqrt{37+22 c+4 c^{2}}$. At large $c$ this gives

$$
\lambda_{1}=10 c=\frac{4^{3}-4}{6} c,
$$

$$
\lambda_{2}=2 c=2 \frac{2^{3}-2}{6} c .
$$

Level 5: A (not orthonormal) basis is $\left\{L_{-5}|0\rangle\right.$, $\left.L_{-3} L_{-2}|0\rangle\right\}$. The matrix representation of $\tilde{Q}^{(1)}$ in this basis is

$$
\left(\begin{array}{cc}
20 c+50 & 9 c+18 \\
30 & 5 c+50
\end{array}\right)
$$

with eigenvalues $\lambda_{1 / 2}=\frac{100+25 c \pm \sqrt{45\left(48+24 c+5 c^{2}\right)}}{2}$. At large $c$ this gives

$$
\begin{gathered}
\lambda_{1}=20 c=\frac{5^{3}-5}{6} c, \\
\lambda_{2}=5 c=\left(\frac{3^{3}-3}{6}+\frac{2^{3}-2}{6}\right) c .
\end{gathered}
$$

Level 6: A (not orthonormal) basis is $\left\{L_{-6}|0\rangle\right.$, $\left.L_{-4} L_{-2}|0\rangle, L_{-3} L_{-3}|0\rangle, L_{-2} L_{-2} L_{-2}|0\rangle\right\}$. The matrix representation of $\tilde{Q}^{(1)}$ in this basis is

$$
\left(\begin{array}{cccc}
35 c+88 & 12 c & 36 c+72 & 48 c+48 \\
36 & 11 c+42 & 108 & 18 c+132 \\
18 & 24 & 8 c+52 & 48 \\
0 & 11 & 0 & 3 c+48
\end{array}\right)
$$


At large $c$ the four eigenvalues are

$$
\begin{gathered}
\lambda_{1}=35 c=\frac{6^{3}-6}{6} c, \\
\lambda_{2}=11 c=\left(\frac{4^{3}-4}{6}+\frac{2^{3}-2}{6}\right) c, \\
\lambda_{3}=8 c=2 \frac{3^{3}-3}{6} c, \\
\lambda_{4}=3 c=3 \frac{2^{3}-2}{6} c,
\end{gathered}
$$

\section{Global $Q$ character}

By the global character we denote the object that follows from taking the trace over states in the submodule corresponding to the global subalgebra of the full Virasoro algebra, i.e., $s l_{2} \subset \operatorname{Vir}_{c}$. The $s l_{2}$ submodule $\left.[p]\right|_{s l_{2}}$ of the primary module $[p]$ is built upon states of the form $L_{-1}^{k}|p\rangle, k \in \mathbb{N}$.

We also have to restrict $Q$ to the parts that act within this global submodule. In the present case this means we only keep the terms that include $s l_{2}$ generators,

$$
\begin{aligned}
Q_{s l_{2}} & \equiv 2 \hbar^{\prime}\left(2 c L_{-1}^{\prime} L_{1}^{\prime}+L_{0}^{2}-\frac{c-1}{12} L_{0}\right) \\
& \equiv Q_{s l_{2}}^{(1)}+2 \hbar^{\prime} L_{0}^{2}-2 \hbar^{\prime} \frac{c-1}{12} L_{0} .
\end{aligned}
$$

All the terms that we omitted act as

$$
\begin{aligned}
& 2 \hbar^{\prime} c L_{-m}^{\prime} L_{m}^{\prime} L_{-1}^{\prime k}|p\rangle \\
& \quad=2 \hbar^{\prime} \frac{(m+1) !}{c^{\frac{m-1}{2}}}\left(\begin{array}{c}
k \\
k-m
\end{array}\right)\left(\frac{k-m}{m+1}+p\right) L_{-m}^{\prime} L_{-1}^{\prime k-m}|p\rangle \\
& \left.\equiv Q_{k, m, p} L_{-m}^{\prime} L_{-1}^{k-m}|p\rangle \notin[p]\right|_{s l_{2}} \quad \text { for } m>1 .
\end{aligned}
$$

They do not act within the global subrepresentation and hence have to be omitted.

The $Q_{s l_{2}}^{(1)}$ eigenvalues of a global state $L_{-1}^{k}|p\rangle$ are exactly the one in (29) with $h_{\psi}=p$, so

$$
Q_{s l_{2}}^{(1)} L_{-1}^{k}|p\rangle=4 \hbar^{\prime}\left(k^{2}+(2 p-1) k\right) L_{-1}^{k}|p\rangle .
$$

It follows immediately that the $Q_{s l_{2}}^{(1)}$ character in the global module $\left.[p]\right|_{s l_{2}}$ is given by

$$
\operatorname{Tr}_{[p]_{s l_{2}}} y_{1}^{Q_{s l_{2}}^{(1)}}=\left(\sum_{k=0}^{\infty} y_{1}^{4 \hbar^{\prime}\left(k^{2}+(2 p-1) k\right)}\right)
$$

The $L_{0}$ eigenvalues of the previous states are simply $(p+k)$. The full $Q_{s l_{2}}$ character is, hence, given by

$\operatorname{Tr}_{[p]_{s l_{2}}} q^{Q_{s l_{2}}}=\sum_{k=0}^{\infty} y_{1}^{2 \hbar^{\prime}\left(2 k^{2}+(2 p-1) 2 k+(p+k)^{2}-\frac{c-1}{12}(p+k)\right)}$.

We see here that we cannot simply interchange the limit and the summation. This is because the eigenvalues switch sign at $k \sim c$ which completely changes the behavior of the sum. In particular, when $c$ is very large, then $k \lesssim c$ gives huge contributions to the character.

\section{a. Closed forms for global $Q$ characters}

The eigenvalues

$$
\lambda(k)=2 \hbar^{\prime}\left(2 k^{2}+(2 p-1) 2 k+(p+k)^{2}-\frac{c-1}{12}(p+k)\right)
$$

are negative between

$$
k_{ \pm}=\frac{c+23-72 p \pm \sqrt{529+46 c+c^{2}-3456 p+3456 p^{2}}}{72} .
$$

Hence, for large $c \gg p, \lambda$ is negative between $k_{-}<k_{0}=0$ and $k_{+} \approx \frac{c}{36}$. The minimum lies at $k_{\min }=$ $\frac{23+c-72 p}{72} \approx \frac{c}{72}$, with a minimal value of

$$
\lambda\left(k_{\min }\right)=-\hbar \frac{c^{2}+46 c+3456 p^{2}-3456 p+529}{864} \approx-\frac{\hbar c^{2}}{864} .
$$

The sum (34) for $k<k_{+}$can be approximated by an integral for $\mu \gg 1 / c$ and $c \gg p$. It gives

$\sum_{k=0}^{k_{+}} y_{1}^{\lambda(k)} \approx \sqrt{\frac{\pi c}{18 \mu_{1}}} \exp \left[\mu_{1}\left(\frac{c}{288}+\frac{23}{144}+\frac{12 p(p-1)+\frac{529}{288}}{c}\right)\right]$.

It is obvious that the contribution to the global character by negative eigenvalues is huge.

Note that in case of higher charges one also needs to consider the first subleading contribution from the charge. Higher order corrections in $1 / c$ are not needed to render the sum finite. The global character looks like (B21) but dressed with coefficients that follow from (41).

Note further that it seems reasonable that the global character behaves somehow nice under modular transformation, since their form looks much like the well known 
$\theta$ functions. If we for example take the primary to be $p=\frac{1}{2}$, then (B20) is given by $\frac{1}{2}\left(\theta_{3}(\tau)+1\right)$ with $\tau=i \frac{24 \mu_{1}}{c \pi}$ and

$$
\theta_{3}(\tau)=\sum_{n \in \mathbb{Z}} q^{\frac{n^{2}}{2}}
$$

Or for $p=1$ it can be written as $\frac{q^{1 / 4}}{2} \theta_{2}(\tau)$ with

$$
\theta_{2}(\tau)=\sum_{n \in \mathbb{Z}} q^{\frac{\left(n+\frac{1}{2}\right)^{2}}{2}}
$$

It is possible to write the general global character in any primary module as the generalized $\theta$-function given by

$$
\begin{aligned}
\theta[\alpha, \beta](\tau, z)= & \sum_{n \in \mathbb{Z}} q^{\frac{1}{2}(n+\alpha)^{2}} e^{2 \pi i(n+\alpha)(z+\beta)} \\
= & \frac{\eta(\tau) e^{2 \pi i \alpha(z+\beta)}}{q^{\frac{1}{24}-\alpha^{2}}} \prod_{n=1}^{\infty}\left(1+q^{n+\alpha-\frac{1}{2}} e^{2 \pi i(z+\beta)}\right) \\
& \times\left(1+q^{n-\alpha-\frac{1}{2}} e^{-2 \pi i(z+\beta)}\right),
\end{aligned}
$$

which behaves under modular transformations as

$$
\begin{aligned}
& \theta[\alpha, \beta](\tau+1, z)=e^{-i \pi \alpha(\alpha-1)} \theta\left[\alpha, \alpha+\beta-\frac{1}{2}\right](\tau, z), \\
& \theta[\alpha, \beta]\left(-\frac{1}{\tau}, \frac{z}{\tau}\right)=\sqrt{-i \tau} e^{2 \pi i \alpha \beta+i \pi \pi^{\frac{z^{2}}{\tau}}} \theta[\beta,-\alpha](\tau, z) .
\end{aligned}
$$

\section{APPENDIX C: SADDLE}

We want to evaluate by saddle the following integral:

$$
\int d n \exp \left(A(l) n^{1 / 2 l}-\mu_{l} n\right)
$$

The saddle point equation yields

$$
\begin{aligned}
\frac{A(l)}{2 l} n_{s}^{\frac{1-2 l}{2 l}}-\mu_{l} & =0 \\
\text { or } n_{s} & =\left(\frac{2 l \mu_{l}}{A(l)}\right)^{\frac{2 l}{1-2 l}} .
\end{aligned}
$$

Plugging back the saddle point into the integral we obtain

$$
\begin{aligned}
\exp \left(A(l) n_{s}^{1 / 2 l}\right) y_{l}^{n_{s}} & =\exp \left(A(l)\left(\frac{2 l \mu_{l}}{A(l)}\right)^{\frac{1}{1-2 l}}\right) y_{l}^{\left.\frac{2 l \mu_{l}}{A(l)}\right)^{\frac{2 l}{1-2 l}}} \\
& =\exp \left(A(l)^{\frac{2 l}{2 l-1}}\left(2 l \mu_{l}\right)^{\frac{1}{1-2 l}}\left(1-(2 l)^{-1}\right)\right) .
\end{aligned}
$$

Thus,

$$
\int d n \exp \left(A(l) n^{1 / 2 l}\right) y_{l}^{n} \approx \exp \left\{\left(1-\frac{1}{2 l}\right) A(l)^{\frac{2 l}{2 l-1}}\left(2 l \mu_{l}\right)^{\frac{1}{1-2 l}}\right\} .
$$

[1] R. Sasaki and I. Yamanaka, Virasoro algebra, vertex operators, quantum sine-gordon and solvable quantum field theories, Adv. Stud. Pure Math. 16, 271 (1988).

[2] T. Eguchi and S.-K. Yang, Deformations of conformal field theories and soliton equations, Phys. Lett. B 224, 373 (1989).

[3] V. V. Bazhanov, S. L. Lukyanov, and A. B. Zamolodchikov, Integrable structure of conformal field theory, quantum $\mathrm{KdV}$ theory and thermodynamic Bethe ansatz, Commun. Math. Phys. 177, 381 (1996).

[4] V. V. Bazhanov, S. L. Lukyanov, and A. B. Zamolodchikov, Spectral determinants for Schrodinger equation and Q operators of conformal field theory, J. Stat. Phys. 102, 567 (2001).

[5] V. V. Bazhanov, S. L. Lukyanov, and A. B. Zamolodchikov, Higher level eigenvalues of Q operators and Schroedinger equation, Adv. Theor. Math. Phys. 7, 711 (2003).

[6] S. Negro, Integrable structures in quantum field theory, J. Phys. A 49, 323006 (2016).

[7] A. B. Zamolodchikov, Integrals of motion and s-matrix of the (scaled) $t=t c$ ising model with magnetic field, Int. J. Mod. Phys. A 04, 4235 (1989).
[8] W. Nahm, A semiclassical calculation of the mass spectrum of relativistic strings, Nucl. Phys. B81, 164 (1974).

[9] J. L. Cardy, Operator content of two-dimensional conformally invariant theories, Nucl. Phys. B270, 186 (1986).

[10] P. Kraus and A. Maloney, A cardy formula for three-point coefficients or how the black hole got its spots, J. High Energy Phys. 05 (2017) 160.

[11] D. Das, S. Datta, and S. Pal, Charged structure constants from modularity, J. High Energy Phys. 11 (2017) 183.

[12] E. M. Brehm, D. Das, and S. Datta, Probing thermality beyond the diagonal, Phys. Rev. D 98, 126015 (2018).

[13] B. Eynard, Lectures notes on compact Riemann surfaces, arXiv:1805.06405.

[14] A. Maloney, S. G. Ng, S. F. Ross, and I. Tsiares, Generalized gibbs ensemble and the statistics of $\mathrm{KdV}$ charges in 2D CFT, J. High Energy Phys. 03 (2019) 75.

[15] A. Dymarsky and K. Pavlenko, Generalized Gibbs Ensemble of $2 \mathrm{~d}$ CFTs at large central charge in the thermodynamic limit, J. High Energy Phys. 01 (2019) 98.

[16] A. Maloney, S. Ng, S. F. Ross, and I. Tsiares, Thermal correlation functions of $\mathrm{KdV}$ charges in 2D CFT, J. High Energy Phys. 02 (2019) 044. 
[17] A. Dymarsky and K. Pavlenko, Exact generalized partition function of 2D CFTs at large central charge, J. High Energy Phys. 05 (2019) 077.

[18] H. Dorn and G. Jorjadze, Operator approach to boundary liouville theory, Ann. Phys. (Amsterdam) 323, 2799 (2008).

[19] D. Fioravanti and M. Rossi, Exact conserved quantities on the cylinder 1: Conformal case, J. High Energy Phys. 07 (2003) 031.

[20] A. L. Fitzpatrick, J. Kaplan, M. T. Walters, and J. Wang, Hawking from Catalan, J. High Energy Phys. 05 (2016) 069.

[21] G. H. Hardy and S. Ramanujan, Asymptotic formulaæ in combinatory analysis, Proc. London Math. Soc. s2-17, 75 (1918).

[22] T. Hartman, C. A. Keller, and B. Stoica, Universal spectrum of $2 \mathrm{~d}$ conformal field theory in the large c limit, J. High Energy Phys. 09 (2014) 118.
[23] M. Srednicki, Thermal fluctuations in quantized chaotic systems, J. Phys. A 29, L75 (1996).

[24] J. M. Deutsch, Quantum statistical mechanics in a closed system, Phys. Rev. A 43, 2046 (1991).

[25] M. Rigol, V. Dunjko, and M. Olshanii, Thermalization and its mechanism for generic isolated quantum systems, Nature (London) 452, 854 (2008).

[26] D. A. Roberts and D. Stanford, Two-Dimensional Conformal Field Theory and the Butterfly Effect, Phys. Rev. Lett. 115, 131603 (2015).

[27] W.-Z. Guo, F.-L. Lin, and J. Zhang, Note on ETH of descendant states in 2D CFT, J. High Energy Phys. 01 (2019) 152.

[28] S. Chawla, Kdv charges in 2d free bosons (unpublished).

[29] J. de Boer and D. Engelhardt, Remarks on thermalization in 2D CFT, Phys. Rev. D 94, 126019 (2016). 